\title{
A FAST AND ACCURATE NUMERICAL SCHEME FOR THE PRIMITIVE EQUATIONS OF THE ATMOSPHERE*
}

\author{
JIE SHEN ${ }^{\dagger}$ AND SHOUHONG WANG W $^{\ddagger}$
}

\begin{abstract}
We present a fast and accurate numerical scheme for the approximation of the primitive equations of the atmosphere. The temporal variable is discretized by using a special semiimplicit scheme which requires only to solve a Helmholtz equation and a nonlocal Stokes problem at each time step; the spatial variables are discretized by a spectral-Galerkin procedure with the horizontal components of vectorial spherical harmonics for the horizontal variables and Legendre or Chebyshev polynomials for the vertical variable. The new scheme has two distinct features: (i) it is unconditionally stable given fixed physical parameters, and (ii) the Helmholtz equation and the nonlocal Stokes problem which need to be solved at each time step can be decomposed into a sequence of one-dimensional equations (in the vertical variable) which can be solved by a spectral-Galerkin method with optimal computational complexity.
\end{abstract}

Key words. primitive equations of the atmosphere, spectral-Galerkin, semi-implicit time discretization, unconditional stability

AMS subject classifications. 35Q35, 65M70, 86-08, 86A10

PII. S0036142997324770

1. Introduction. We consider in this article numerical approximations of the primitive equations of the atmosphere, hereafter abbreviated PEs. Physical laws governing the motion and states of the atmosphere can be described by the general equations of hydrodynamics and thermodynamics. However, these equations are far too complicated to be investigated both analytically and numerically; simplifications from both the physical and mathematical points of view are thus inevitable. The PEs are obtained from these general equations with the hydrostatic assumption; see $[28,21,14]$ for more detailed explanations. This assumption is legitimate to the first order since the ratio between the vertical and horizontal scales is very small for the large-scale atmosphere.

The PEs are considered the core equations of the atmosphere. Although the PEs have been studied intensively, many important mathematical and numerical issues related to the PEs still remain to be addressed. We refer to $[14,27]$ for some recent mathematical analysis of PEs. The dynamics of the atmosphere and climate are extremely rich and complicated, and their study involves many aspects of modeling, mathematical and numerical analysis, and numerical simulations. We refer interested readers to [26] for an up-to-date survey of problems and issues in climate sciences.

Although the latest version of the community climate model CCM3 developed by the National Center for Atmospheric Research in Boulder, Colorado was built upon the PEs with detailed physical parameterizations, it is still of interest to develop a relatively simple general circulation model (GCM) using the PEs with simplified

${ }^{*}$ Received by the editors July 18, 1997; accepted for publication (in revised form) July 28, 1998; published electronically March 23, 1999.

http://www.siam.org/journals/sinum/36-3/32477.html

$\dagger$ Department of Mathematics, Penn State University, 322 McAllister Bldg., University Park, PA 16802 (shen_j@math.psu.edu). This author was partially supported by NSF grants DMS-9623020 and DMS-9706951.

${ }^{\ddagger}$ Department of Mathematics, Indiana University, Rawles Hall 115, Bloomington, IN 47405 (showang@indiana.edu, http://php.indiana.edu/ showang/). This author was partially supported by Office of Naval Research grant NAVY-N00014-96-1-0425 and National Science Foundation grant NSF-DMS-9623071. 
physical assumptions to study the essential dynamics of the atmosphere and climate. On the other hand, further simplified models, such as quasi-geostrophic models, are frequently used to study some important climate regimes and phenomena. The availability of a simple GCM model may be used to justify some features of these further simplified models. Therefore, as a first step toward our long-range objective of better understanding the phenomena of atmosphere and climate, in particular the large-scale atmospheric variability on time scales of days to years, we will construct a fast and accurate numerical scheme for the PEs with simplified physical assumptions.

Although there have been numerous numerical studies done for the PEs (see for instance $[12,10,25,9,20]$ and a nice review in [13]), there are some essential differences between our scheme and those of others:

- We study directly the vectorial form of the PEs instead of using the divergence and vorticity form of the PEs which are derived from differentiating the vectorial PEs and which need to be supplemented with subtle boundary conditions.

- With a special time discretization scheme, we need only to solve a standard Helmholtz equation and a nonlocal Stokes problem at each time step. Using vectorial spherical harmonics, we are able to decompose the PEs into a sequence of one-dimensional (in the vertical direction) two points boundary value problems which can be solved by a new fast spectral-Galerkin method $[23,24]$ using Legendre or Chebyshev polynomials.

- The new scheme is shown to be unconditionally stable for fixed physical parameters, i.e., the allowable time step is related only to the physical parameters, but is not restricted by the spatial resolution.

Of course, we use simplified physical assumptions to handle the surface boundary layers, but this is legitimate in the sense that they retain the essential dynamics of the atmosphere and climate. Also, we do not take into account the humidity. However, it is obvious that the humidity equation can be easily incorporated into our scheme and that the more sophisticated (or realistic) viscosity involving the Richardson number does not introduce any essential difficulty so that it can be handled with slight modifications.

This article is organized as follows. The nondimensional form of the PEs is described in section 2 . In section 3 , we propose two special semi-implicit time discretization schemes of the PEs. In section 4, we present a fast spectral-Galerkin method for the Helmholtz equation and the nonlocal Stokes problem. Some concluding remarks are given in section 5. For readers' convenience, we provide a brief derivation of the nondimensional form of the PEs in Appendix A, and we also gather some basic facts about the scalar and vectorial spherical harmonics in Appendix B.

2. The primitive equations. With proper scaling (see Appendix A and [14, 15] for more details), the nondimensional PEs read

$$
\begin{aligned}
& \frac{\partial v}{\partial t}+\nabla_{v} v-W(v) \frac{\partial v}{\partial \eta}+\frac{1}{R o}\left[f k \times v+\nabla \phi_{s}+\nabla \mathcal{M}\left(\frac{T}{K_{2}}\right)\right]+L_{1} v=0 \\
& \alpha\left[\frac{\partial T}{\partial t}+\nabla_{v} T-W(v) \frac{\partial T}{\partial \eta}\right]-\frac{1}{R o} \frac{W(v)}{K_{2}}+L_{2} T=Q \\
& \operatorname{div} \int_{0}^{1} v d \eta=0
\end{aligned}
$$


with the initial condition

$$
u \equiv(v, T)=u_{0}=\left(v_{0}, T_{0}\right) \text { at } t=0,
$$

and the boundary conditions

$$
\left\{\begin{array}{l}
\frac{\partial v}{\partial \eta}=\gamma_{s} v, \quad \omega=0 \text { and } \frac{\partial T}{\partial \eta}=\alpha_{s}\left(T-T_{s}\right) \text { at } \eta=0, \\
\frac{\partial v}{\partial \eta}=0, \quad \omega=0 \text { and } \frac{\partial T}{\partial \eta}=0 \text { at } \eta=1 .
\end{array}\right.
$$

Some explanations are in order.

- The unknown functions are the horizontal velocity $v$, the temperature function $T$, and the surface geopotential function $\phi_{s}$. Note that a function defined only on the surface of the sphere is denoted by adding a subscript $s$ to the function name.

- The domain for the space variables $(\theta, \varphi, \eta)$ is the nondimensional pseudospatial domain

$$
\mathcal{D}=S^{2} \times(0,1)
$$

with $S^{2}$ being the unit sphere. Here $\theta$ stands for the colatitude, $\varphi$ the longitude, and $\eta$ the nondimensional pressure (see Appendix A).

- The operator $\mathcal{M}$, its adjoint (in the $L^{2}$ sense) $\mathcal{M}^{*}$, and the operator $W$ are defined by

$$
\mathcal{M} \psi(\eta)=\int_{0}^{\eta} \psi d \eta^{\prime}, \quad \mathcal{M}^{*} \psi(\eta)=\int_{\eta}^{1} \psi d \eta^{\prime}, \quad W(v)=-\operatorname{div} \mathcal{M}^{*} v
$$

- The two linear elliptic operators $L_{1}$ and $L_{2}$ are defined by

$$
\left\{\begin{array}{l}
L_{1}=-\frac{1}{R e_{1}} \Delta-\frac{1}{R e_{2}} \frac{\partial}{\partial \eta}\left(K_{1} \frac{\partial}{\partial \eta}\right) \\
L_{2}=-\frac{1}{R t_{1}} \Delta-\frac{1}{R t_{2}} \frac{\partial}{\partial \eta}\left(K_{1} \frac{\partial}{\partial \eta}\right) .
\end{array}\right.
$$

- The operators $\nabla, \nabla_{v} \equiv v \cdot \nabla$, div, and $\Delta$ are horizontal differential operators in spherical coordinates on $S^{2}$.

- $\alpha, R o, R e_{1}, R e_{2}, R t_{1}$, and $R t_{2}$ are given positive constants; $K_{1}, K_{2}, \gamma_{s}$, and $\alpha_{s}$ are given positive functions; $f, T_{s}, Q$, and the initial data $u_{0}=\left(v_{0}, T_{0}\right)$ are given functions; $k$ is the unit vector in the vertical direction.

More details on the derivation of the nondimensional PEs and the physical meanings of the various quantities are given in Appendix A.

The mathematical analysis of the PEs, including the existence, uniqueness, and long-term behavior of the solutions, was previously conducted in [14] and [27]. In particular, the authors proved the existence of global (in time) weak solutions for arbitrary $L^{2}$ initial data, as well as the existence and uniqueness of global (in time) strong solutions with small data.

3. Special semi-implicit time discretization schemes. We construct below a special semi-implicit discretization scheme for the PEs. Let $\Delta t$ be the time step, 
$u^{n}=\left(v^{n}, T^{n}\right)$ and $\phi_{s}^{n}$ be the approximate solutions to $(v, T)$ and $\phi_{s}$ at time $t=n \Delta t$. We use $\bar{K}_{1}, \bar{\alpha}_{s}$, and $\bar{\gamma}_{s}$ to denote the following positive numbers:

$$
\left\{\begin{array}{l}
\bar{K}_{1}=\frac{1}{2}\left[\max _{S^{2}} K_{1}+\min _{S^{2}} K_{1}\right], \\
\bar{\gamma}_{s}=\frac{1}{2}\left[\max _{S^{2}} \gamma_{s}+\min _{S^{2}} \gamma_{s}\right] \\
\bar{\alpha}_{s}=\frac{1}{2}\left[\max _{S^{2}} \alpha_{s}+\min _{S^{2}} \alpha_{s}\right]
\end{array}\right.
$$

and we denote by $\bar{L}_{1}$ and $\bar{L}_{2}$ the following two linear constant-coefficient operators associated with the linear operators $L_{1}$ and $L_{2}$ :

$$
\left\{\begin{array}{l}
\bar{L}_{1}=-\frac{1}{R e_{1}} \Delta-\frac{\bar{K}_{1}}{R e_{2}} \frac{\partial^{2}}{\partial \eta^{2}}, \\
\bar{L}_{2}=-\frac{1}{R t_{1}} \Delta-\frac{\bar{K}_{1}}{R t_{2}} \frac{\partial^{2}}{\partial \eta^{2}} .
\end{array}\right.
$$

We can now write our first-order semi-implicit scheme for the PEs (2.1)-(2.5) as follows.

Scheme 3.1. Let $u^{0}=\left(v_{0}, T_{0}\right)$. When $\left(v^{j}, \phi_{s}^{j}, T^{j}\right)(j=0,1, \ldots, n)$ are known, we define $\left(v^{n+1}, \phi_{s}^{n+1}, T^{n+1}\right)$ as the solution of the following linear system:

$$
\begin{aligned}
& \frac{v^{n+1}-v^{n}}{\Delta t}+\frac{1}{R o}\left[f k \times v^{n}+\nabla \phi_{s}^{n+1}+\nabla \mathcal{M}\left(\frac{T^{n}}{K_{2}}\right)\right] \\
& \quad+\nabla_{v^{n}} v^{n}-W\left(v^{n}\right) \frac{\partial v^{n}}{\partial \eta}+\bar{L}_{1} v^{n+1}=\frac{1}{R e_{2}} \frac{\partial}{\partial \eta}\left[\left(K_{1}-\bar{K}_{1}\right) \frac{\partial v^{n}}{\partial \eta}\right], \\
& \alpha\left[\frac{T^{n+1}-T^{n}}{\Delta t}+\nabla_{v^{n}} T^{n}-W\left(v^{n}\right) \frac{\partial T^{n}}{\partial \eta}\right]-\frac{1}{R o} \frac{W\left(v^{n}\right)}{K_{2}} \\
& \quad+\bar{L}_{2} T^{n+1}=Q^{n}+\frac{1}{R t_{2}} \frac{\partial}{\partial \eta}\left[\left(K_{1}-\bar{K}_{1}\right) \frac{\partial T^{n}}{\partial \eta}\right], \\
& \operatorname{div} \int_{0}^{1} v^{n+1} d \eta=0,
\end{aligned}
$$

with the following boundary conditions:

$$
\left\{\begin{array}{l}
\frac{\partial v^{n+1}}{\partial \eta}=\bar{\gamma}_{s} v^{n+1}-\left(\bar{\gamma}_{s}-\gamma_{s}\right) v^{n} \quad \text { for } \eta=0 \\
\frac{\partial T^{n+1}}{\partial \eta}=\bar{\alpha}_{s} T^{n+1}-\left(\bar{\alpha}_{s}-\alpha_{s}\right) T^{n}-\alpha_{s} T_{s} \quad \text { for } \eta=0 \\
\frac{\partial v^{n+1}}{\partial \eta}=0, \quad \frac{\partial T^{n+1}}{\partial \eta}=0 \quad \text { for } \eta=1 .
\end{array}\right.
$$

A few remarks are in order:

- To facilitate the numerical computation, terms involving nonconstant coefficients $K_{1}, \gamma_{s}$, and $\alpha_{s}$ are split up into an implicit part with constant coefficients and an explicit part with nonconstant coefficients. This treatment will enable us to decompose (3.4)-(3.6) into a sequence of one-dimensional problems (see the next section) while preserving the stability of the scheme. 
- $\left(v^{n+1}, \phi_{s}^{n+1}\right)$ and $T^{n+1}$ in Scheme 3.1 are decoupled. More precisely, at each time step, we need only to solve a generalized nonlocal Stokes problem for $\left(v^{n+1}, \phi_{s}^{n+1}\right)$

$$
\left\{\begin{array}{l}
\frac{1}{\Delta t} v^{n+1}+\bar{L}_{1} v^{n+1}+\frac{1}{R o} \nabla \phi_{s}^{n+1}=f_{1}^{n} \text { in } S^{2} \times(0,1), \\
\operatorname{div} \int_{0}^{1} v^{n+1} d \eta=0 \text { in } S^{2} \\
\int_{S^{2}} \phi_{s}^{n+1} d A=0
\end{array}\right.
$$

and a standard Helmholtz equation for $T^{n+1}$

$$
\frac{\alpha}{\Delta t} T^{n+1}+\bar{L}_{2} T^{n+1}=f_{2}^{n} \text { in } S^{2} \times(0,1),
$$

subject to the boundary conditions in (3.6) and with $f_{1}^{n}$ and $f_{2}^{n}$ depending only on functions of the previous time step. Since the solution $\left(v^{n+1}, \phi_{s}^{n+1}\right)$ of the first two equations in (3.7) is unique only up to a constant for $\phi_{s}^{n+1}$, we added the constraint, the third equation in (3.7), for $\phi_{s}^{n+1}$ to determine uniquely this function.

Scheme 3.1 is only first-order accurate in time. However, one can also construct a second-order semi-implicit scheme with similar properties. To this end, we denote

$$
\begin{aligned}
R H S 1^{n}= & -\nabla_{v^{n}} v^{n}+W\left(v^{n}\right) \frac{\partial v^{n}}{\partial \eta}-\frac{1}{R o}\left[f k \times v^{n}+\nabla \mathcal{M}\left(\frac{T^{n}}{K_{2}}\right)\right], \\
R H S 2^{n}= & Q^{n}+\frac{1}{R t_{2}} \frac{\partial}{\partial \eta}\left[\left(K_{1}-\bar{K}_{1}\right) \frac{\partial T^{n}}{\partial \eta}\right] \\
& \quad-\alpha\left[\nabla_{v^{n}} T^{n}-W\left(v^{n}\right) \frac{\partial T^{n}}{\partial \eta}\right]+\frac{1}{R o} \frac{W\left(v^{n}\right)}{K_{2}}, \\
B D 1^{n}= & \left(\bar{\gamma}_{s}-\gamma_{s}\right) v^{n}, \\
B D 2^{n}= & \left(\bar{\alpha}_{s}-\alpha_{s}\right) T^{n}+\alpha_{s} T_{s} .
\end{aligned}
$$

Then, a second-order semi-implicit scheme can be written as follows.

\section{Scheme 3.2.}

$$
\begin{aligned}
& \frac{1}{2 \Delta t}\left(3 v^{n+1}-4 v^{n}+v^{n-1}\right)+\bar{L}_{1} v^{n+1}+\frac{1}{R o} \nabla \phi_{s}^{n+1} \\
& =\left(2 R H S 1^{n}-R H S 1^{n-1}\right), \\
& \frac{1}{2 \Delta t}\left(3 T^{n+1}-4 T^{n}+T^{n-1}\right)+\bar{L}_{2} T^{n+1}=\left(2 R H S 2^{n}-R H S 2^{n-1}\right), \\
& \operatorname{div} \int_{0}^{1} v^{n+1} d \eta=0,
\end{aligned}
$$

with the boundary conditions

$$
\begin{cases}\frac{\partial v^{n+1}}{\partial \eta}=\bar{\gamma}_{s} v^{n+1}-\left(2 B D 1^{n}-B D 1^{n-1}\right) & \text { for } \eta=0 \\ \frac{\partial T^{n+1}}{\partial \eta}=\bar{\alpha}_{s} T^{n+1}-\left(2 B D 2^{n}-B D 2^{n-1}\right) & \text { for } \eta=0 \\ \frac{\partial v^{n+1}}{\partial \eta}=0, \quad \frac{\partial T^{n+1}}{\partial \eta}=0 \quad \text { for } \eta=1 . & \end{cases}
$$


It is obvious that at each time step Scheme 3.2 also leads to a nonlocal Stokes problem and a Helmholtz equation. We emphasize that for fixed physical parameters these schemes are unconditionally stable. More precisely, it can be shown and is verified by preliminary numerical results that for fixed physical parameters, and assuming the existence of the strong solution in the time interval $(0, \tau]$, there exists $\Delta t_{c}$ such that for all $\Delta t \leq \Delta t_{c}$ and $n \leq \frac{\tau}{\Delta t}-1$,

$$
\left\|v^{n+1}-v((n+1) \Delta t)\right\|_{L^{2}(\mathcal{D})}+\left\|T^{n+1}-T((n+1) \Delta t)\right\|_{L^{2}(\mathcal{D})} \leq C(\Delta t)^{\alpha},
$$

where $\alpha=1$ for Scheme 3.1 and $\alpha=2$ for Scheme 3.2. Therefore, when Schemes 3.1 and 3.2 are further approximated by any appropriate spatial discretization, the allowable time step will be related only to the physical parameters, but not restricted by the spatial resolution.

4. A fast and accurate spectral approximation of the nonlocal Stokes problem. We recall that after time discretization, one needs only to solve a nonlocal Stokes problem and a Helmholtz equation. This section is devoted to constructing a fast and accurate approximation to these equations.

Since the numerical approximation of the Helmholtz equation is standard, we consider in this section only the numerical approximation for the generalized nonlocal Stokes problem

$$
\left\{\begin{array}{l}
a v-b \Delta v-e \frac{\partial^{2} v}{\partial \eta^{2}}+\nabla \phi_{s}=f \text { in } S^{2} \times(0,1), \\
\operatorname{div} \int_{0}^{1} v d \eta=0 \text { in } S^{2} \\
\int_{S^{2}} \phi_{s} d A=0 .
\end{array}\right.
$$

The boundary conditions for (4.1) are the same as those given for the horizontal velocity in the primitive equations, namely,

$$
\left\{\begin{array}{l}
\frac{\partial v}{\partial \eta}=0 \text { at } \eta=1, \\
\frac{\partial v}{\partial \eta}=\gamma v-v_{s} \text { at } \eta=0 .
\end{array}\right.
$$

Here, $a b, e$, and $\gamma$ are given positive constants; $f$ and $v_{s}$ are given functions.

It is proved in [14] that there exists a unique solution $\left(v, \phi_{s}\right)$ to the nonlocal Stokes boundary value problem (4.1)-(4.2). In this section, we propose a spectral approximation for (4.1)-(4.2).

4.1. Decomposition via spherical harmonics and error analysis. Let $Y_{\ell, m}$ $(\ell=0,1, \ldots,|m| \leq \ell)$ be the usual scalar spherical harmonics (see Appendix B for its definition). Then, $C_{\ell, m}=\lambda_{l}^{-1 / 2} \operatorname{curl} Y_{\ell, m}$ and $G_{\ell, m}=\lambda_{l}^{-1 / 2} \nabla Y_{\ell, m}$ form the basis of the horizontal components of the vectorial spherical harmonics (see Appendix B for details). Hence, we have formally the following spectral expansion of the horizontal vector field $v$ and external forcing $f$ :

$$
v=\sum_{\substack{\ell=1 \\|m| \leq \ell}}^{\infty}\left[v_{g}^{\ell, m}(\eta) G_{\ell, m}(\theta, \varphi)+v_{c}^{\ell, m}(\eta) C_{\ell, m}(\theta, \varphi)\right],
$$


where $v_{g}^{\ell, m}(\eta), v_{c}^{\ell, m}(\eta) \in \mathbb{C}^{1}$ are complex, continuously differentiable functions of $\eta$. Note that the constant terms (corresponding to $\ell=0$ ) are excluded in the expansions to eliminate the arbitrary constants. The vector field $v$ is naturally decomposed into the nonrotational part $v_{g}$ and nondivergent part $v_{c}$ with

$$
\left\{\begin{array}{l}
v_{g}=\sum_{\substack{\ell=1 \\
|m| \leq \ell}}^{\infty} v_{g}^{\ell, m}(\eta) G_{\ell, m}(\theta, \varphi), \\
v_{c}=\sum_{\substack{\ell=1 \\
|m| \leq \ell}}^{\infty} v_{c}^{\ell, m}(\eta) C_{\ell, m}(\theta, \varphi) .
\end{array}\right.
$$

To fix the notation, we expand also the external forcing term $f=f_{g}+f_{c}$ with

$$
\left\{\begin{array}{l}
f_{g}=\sum_{\substack{\ell=1 \\
|m| \leq \ell}}^{\infty} f_{g}^{\ell, m}(\eta) G_{\ell, m}(\theta, \varphi), \\
f_{c}=\sum_{\substack{\ell=1 \\
|m| \leq \ell}}^{\infty} f_{c}^{\ell, m}(\eta) C_{\ell, m}(\theta, \varphi) .
\end{array}\right.
$$

Since the geopotential function $\phi_{s}$ is independent of $\eta$, we have

$$
\phi_{s}(\theta, \varphi)=\sum_{\substack{\ell=1 \\|m| \leq \ell}}^{\infty} \phi_{s}^{\ell, m} Y_{\ell, m}(\theta, \varphi) .
$$

We also write

$$
v_{s}=\sum_{\substack{\ell=1 \\|m| \leq \ell}}^{\infty}\left[v_{s g}^{\ell, m} G_{\ell, m}+v_{s c}^{\ell, m} C_{\ell, m}\right] .
$$

Now inserting these expansions into (4.1)-(4.2), we find that the nonlocal Stokes problem (4.1)-(4.2) is decomposed into two sets of one-dimensional boundary value problems:

$$
\begin{aligned}
& \left\{\begin{array}{l}
\left(a+b \lambda_{\ell}\right) v_{c}^{\ell, m}-e \frac{d^{2} v_{c}^{\ell, m}}{d \eta^{2}}=f_{c}^{\ell, m}, \\
\left(v_{c}^{\ell, m}\right)^{\prime}(1)=0,\left(v_{c}^{\ell, m}\right)^{\prime}(0)=\gamma v_{c}^{\ell, m}(0)-v_{s c}^{\ell, m} ;
\end{array}\right. \\
& \left\{\begin{array}{l}
\left(a+b \lambda_{\ell}\right) v_{g}^{\ell, m}-e \frac{d^{2} v_{g}^{\ell, m}}{d \eta^{2}}+\phi_{s}^{\ell, m}=f_{g}^{\ell, m}, \\
\int_{0}^{1} v_{g}^{\ell, m}\left(\eta^{\prime}\right) d \eta^{\prime}=0, \\
\left(v_{g}^{\ell, m}\right)^{\prime}(1)=0,\left(v_{g}^{\ell, m}\right)^{\prime}(0)=\gamma v_{g}^{\ell, m}(0)-v_{s g}^{\ell, m} .
\end{array}\right.
\end{aligned}
$$

Then, we have only to solve the following two prototypical problems. 
The first is a standard one-dimensional Helmholtz equation

$$
\left\{\begin{array}{l}
\alpha u-u_{x x}=f(x), \quad x \in(-1,1) \equiv I, \\
a_{ \pm} u( \pm 1)+b_{ \pm} u_{x}( \pm 1)=\beta_{ \pm},
\end{array}\right.
$$

and the second is a nonlocal one-dimensional elliptic equation

$$
\left\{\begin{array}{l}
\alpha u-u_{x x}+\gamma=f(x), \quad x \in I, \\
\int_{-1}^{1} u(x) d x=0 \\
a_{ \pm} u( \pm 1)+b_{ \pm} u_{x}( \pm 1)=\beta_{ \pm} .
\end{array}\right.
$$

Since the nonhomogeneous boundary conditions $\left(\beta_{ \pm} \neq 0\right)$ can be easily handled by subtracting from the solution a simple function satisfying the boundary conditions, we shall consider only the case with $\beta_{ \pm}=0$.

It is well known (see, for instance, [8]) that (4.7) has a unique solution if

$$
a_{-}^{2}+b_{-}^{2} \neq 0, a_{-} b_{-} \leq 0 ; a_{+}^{2}+b_{+}^{2} \neq 0, a_{+} b_{+} \geq 0 .
$$

The above is verified by (4.5) since $\gamma>0$. Similarly, we can easily show that (4.8) has a unique solution under the above conditions by eliminating $\gamma$ and the integral condition in (4.8).

Let us first consider the approximation of (4.7).

Let $P_{N}$ be the space of all polynomials of degree $\leq N$ and

$$
X_{N}=\left\{u \in P_{N}: a_{ \pm} u( \pm 1)+b_{ \pm} u_{x}( \pm 1)=0\right\} .
$$

We denote

$$
(u, v)_{w}=\int_{-1}^{1} u v w d x
$$

where $w \equiv 1$ in the Legendre case and $w=\left(1-x^{2}\right)^{-1 / 2}$ in the Chebyshev case. Then the spectral-Galerkin approximation for (4.7) (with $\beta_{ \pm}=0$ ) is as follows.

Find $u_{N} \in X_{N}$ such that

$$
\alpha\left(u_{N}, v\right)_{w}-\left(u_{N}^{\prime \prime}, v\right)_{w}=(f, v)_{w} \quad \forall v \in X_{N} .
$$

Remark 4.1. In actual implementation (see section 4.2 below), $f$ in (4.9) should be replaced by $I_{N} f \in P_{N}$ which is the polynomial interpolation of $f$ at the Legendre or Chebyshev Gauss-Lobatto points. We note that in the Legendre case, the scheme can be accelerated by using the interpolation operator $I_{N}$ based on the Chebyshev GaussLobatto points thanks to the availability of a fast transform between the Chebyshev and Legendre polynomials (cf. [1] and [24]).

Hereafter, $\|\cdot\|_{s, \omega}$ is used to denote the weighted Sobolev norm in $H_{\omega}^{s}(I)$ (cf. [5]), and the expression " $A_{N} \lesssim B_{N}$ " means that there exists a constant $C$ independent of $N$ such that " $A_{N} \leq C B_{N}$."

It is evident that the approximation (4.9) is spectrally accurate. More precisely, we have the following result.

THEOREM 4.1. Let $u$ and $u_{N}$ be, respectively, the solution of (4.7) with $\beta_{ \pm}=0$ and (4.9) with $\omega \equiv 1$. Then

$$
\left\|u-u_{N}\right\|_{1} \lesssim N^{1-s}\|f\|_{s-2} \forall s \geq 2 .
$$


Remark 4.2. Here and below, we establish only the error estimates for the case $\omega \equiv 1$. These results can be extended in a straightforward manner to the case of Chebyshev weight with Dirichlet boundary conditions, i.e., $b_{ \pm}=0$ in (4.7)-(4.8). We believe that similar results hold for the case of Chebyshev weight with mixed boundary conditions; however, the actual proof is much more involved and hence will not be addressed here.

The following technical lemma is needed for the proof of Theorem 4.1. Note that the result in Lemma 4.1 is well known in the special case when $b_{ \pm}=0$ (see [5]).

LEMMA 4.1. Let

$$
X=\left\{u \in H_{\omega}^{2}(I): a_{ \pm} u( \pm 1)+b_{ \pm} u_{x}( \pm 1)=0\right\}, \quad X_{N}=X \cap P_{N} .
$$

We have

$$
\inf _{\phi_{N} \in X_{N}}\left\|\phi-\phi_{N}\right\|_{\nu, \omega} \lesssim N^{\nu-s}\|\phi\|_{s, w} \forall \phi \in X \cap H_{\omega}^{s}(I), s \geq 2,0 \leq \nu \leq 2 .
$$

Proof. Let $H(1 ; x)$ be the Hermite polynomial (of degree 3 ) such that

$$
H(1 ; 1)=1, H^{\prime}(1 ; 1)=0, H(1 ;-1)=H^{\prime}(1 ;-1)=0,
$$

and let $\hat{H}(1 ; x)$ be such that

$$
\hat{H}(1 ; 1)=0, \hat{H}^{\prime}(1 ; 1)=1, \hat{H}(1 ;-1)=\hat{H}^{\prime}(1 ;-1)=0 .
$$

Similarly, we define $H(-1 ; x)$ and $\hat{H}(-1 ; x)$. Then for $\phi \in X \cap H_{\omega}^{s}(I)$, we set

$$
\begin{gathered}
\widetilde{\phi}(x)=\phi(x)-\phi(1) H(1 ; x)-\phi(-1) H(-1 ; x) \\
-\phi^{\prime}(1) \hat{H}(1, x)-\phi^{\prime}(-1) \hat{H}(-1 ; x) .
\end{gathered}
$$

By construction, we have

$$
\widetilde{\phi}( \pm 1)=\widetilde{\phi}^{\prime}( \pm 1)=0 \quad \text { and consequently } \quad \widetilde{\phi} \in H_{0, \omega}^{2}(I) .
$$

Furthermore, we derive from (4.10) that

$$
\begin{aligned}
\|\widetilde{\phi}\|_{s, \omega} & \lesssim\|\phi\|_{s, \omega}+|\phi(1)|+|\phi(-1)|+\left|\phi^{\prime}(1)\right|+\left|\phi^{\prime}(-1)\right| \\
& \lesssim\|\phi\|_{s, \omega} \forall s \geq 2 .
\end{aligned}
$$

Let $\Pi_{N}^{2,0}$ be the orthogonal projector from $H_{0, \omega}^{2}(I)$ onto $H_{0, \omega}^{2}(I) \cap P_{N}$ with respect to the inner product $\left(u^{\prime \prime}, v^{\prime \prime}\right)_{\omega}$. It is proven in [3] that

$$
\left\|\widetilde{\phi}-\Pi_{N}^{2,0} \widetilde{\phi}\right\|_{\nu, \omega} \lesssim N^{\nu-s}\|\widetilde{\phi}\|_{s, \omega} \quad \forall \widetilde{\phi} \in H_{0, \omega}^{2}(I) \cap H_{\omega}^{s}(I), 0 \leq \nu \leq 2, s \geq 2 .
$$

We now define

$$
\begin{aligned}
B_{N} \phi= & \Pi_{N}^{2,0} \widetilde{\phi}+\phi(1) H(1 ; x)+\phi(-1) H(-1 ; x) \\
& +\phi^{\prime}(1) \hat{H}(1 ; x)+\phi^{\prime}(-1) \hat{H}(-1 ; x) .
\end{aligned}
$$

Then by (4.10), we have

$$
\begin{aligned}
& \phi-B_{N} \phi=\widetilde{\phi}-\Pi_{N}^{2,0} \widetilde{\phi}, \\
& \left(B_{N} \phi\right)( \pm 1)=\phi( \pm 1),\left(B_{N} \phi\right)^{\prime}( \pm 1)=\phi^{\prime}( \pm 1) .
\end{aligned}
$$


Hence, $B_{N} \phi \in X_{N}$. Therefore, thanks to (4.11) and (4.12), we have

$$
\begin{aligned}
\inf _{\phi_{N} \in X_{N}}\left\|\phi-\phi_{N}\right\|_{\nu, \omega} & \leq\left\|\phi-B_{N} \phi\right\|_{\nu, \omega}=\left\|\widetilde{\phi}-\Pi_{N}^{2,0} \widetilde{\phi}\right\|_{\nu, \omega} \\
& \lesssim N^{\nu-s}\|\widetilde{\phi}\|_{s, \omega} \lesssim N^{\nu-s}\|\phi\|_{s, \omega} \quad \forall s \geq 2 .
\end{aligned}
$$

Proof of Theorem 4.1. From (4.7) and (4.9), we find (cf. [6] for the Chebyshev case)

$$
\begin{aligned}
\left\|u-u_{N}\right\|_{1}^{2} & \lesssim \alpha\left(u-u_{N}, u-u_{N}\right)-\left(\left(u-u_{N}\right)^{\prime \prime}, u-u_{N}\right) \\
& =\alpha\left(u-u_{N}, u-B_{N} u\right)-\left(\left(u-u_{N}\right)^{\prime \prime}, u-B_{N} u\right) \\
& \lesssim \alpha\left\|u-u_{N}\right\|\left\|u-B_{N} u\right\|+\left\|u-B_{N} u\right\|_{1}\left\|u-u_{N}\right\|_{1} .
\end{aligned}
$$

Therefore, thanks to (4.14) and the regularity of (4.7), we have

$$
\left\|u-u_{N}\right\|_{1, \omega} \lesssim\left\|u-B_{N} u\right\|_{1, \omega} \lesssim N^{1-s}\|u\|_{s, \omega} \lesssim N^{1-s}\|f\|_{s-2, \omega} .
$$

The proof of Theorem 4.1 is then complete.

We now consider the approximation of (4.8) (with $\beta_{ \pm}=0$ ) whose (weighted) variational formulation is the following: Find $(u, \gamma) \in X \times \mathbb{R}$ (where $X=H_{0, \omega}^{1}$ ) such that

$$
\begin{aligned}
& \alpha(u, v)_{w}-\left(u^{\prime \prime}, v\right)_{w}+\gamma \int_{-1}^{1} v w d x=(f, v)_{w} \forall v \in X, \\
& \int_{-1}^{1} u d x=0 .
\end{aligned}
$$

The spectral-Galerkin approximation for (4.15) is the following: Find $\left(u_{N}, \gamma_{N}\right) \in$ $X_{N} \times \mathbb{R}$ such that

$$
\begin{aligned}
& \alpha\left(u_{N}, v\right)_{w}-\left(u_{N}^{\prime \prime}, v\right)_{w}+\gamma_{N} \int_{-1}^{1} v w d x=(f, v)_{w} \forall v \in X_{N}, \\
& \int_{-1}^{1} u_{N} d x=0 .
\end{aligned}
$$

THEOREM 4.2. Let $(u, \gamma)$ and $\left(u_{N}, \gamma_{N}\right)$ be, respectively, the solution of (4.15) and (4.16) with $\omega \equiv 1$. Then

$$
\left\|u-u_{N}\right\|_{1}+\left|\gamma-\gamma_{N}\right| \lesssim N^{1-s}\|f\|_{s-2} \forall s \geq 2 .
$$

Proof. The formulation (4.15)-(4.16) with $\omega \equiv 1$ is reminiscent of, although much simpler than, a spectral-Galerkin approximation of the Stokes problem (see, for instance, [4]). Thus, we can adopt the abstract setting for the Stokes problem (for the case of the Chebyshev weight, the abstract setting in [2] should be used).

Let us denote

$$
\begin{aligned}
& a(u, v)=\alpha(u, v)-\left(u^{\prime \prime}, v\right), u, v \in X \\
& b(v, q)=q \int_{-1}^{1} v d x, v \in X, q \in \mathbb{R} .
\end{aligned}
$$


Then (4.15) and (4.16) with $\omega \equiv 1$ can be cast into the following mixed formulations, respectively.

Find $(u, \gamma) \in X \times \mathbb{R}$ such that

$$
\begin{aligned}
a(u, v)+b(v, \gamma) & =(f, v)_{\omega} \forall v \in X, \\
b(u, q) & =0 \quad \forall q \in \mathbb{R} ;
\end{aligned}
$$

find $\left(u_{N}, \gamma_{N}\right) \in X_{N} \times \mathbb{R}$ such that

$$
\begin{aligned}
a\left(u_{N}, v\right)+b\left(v, \gamma_{N}\right) & =(f, v)_{\omega} \forall v \in X_{N} \\
b\left(u_{N}, q\right) & =0 \quad \forall q \in \mathbb{R} .
\end{aligned}
$$

Thus, according to the abstract theory for the mixed formulation (see, for instance, $[4]$ ), we need only to prove the following inf-sup condition:

$$
\sup _{v \in X_{N}} \frac{b(v, q)}{\|v\|_{1}} \geq \beta|q| \forall q \in \mathbb{R} .
$$

Let us choose $a, b$ such that

$$
\phi_{0}(x)=L_{0}(x)+a L_{1}(x)+b L_{2}(x) \in X,
$$

where $L_{i}(x)$ is the $i$ th degree Legendre polynomial. Then, by taking $v=\phi_{0}$ or $-\phi_{0}$ in (4.20), the condition (4.20) is satisfied with

$$
\beta=\frac{\left|\int_{-1}^{1} \phi_{0} d x\right|}{\left\|\phi_{0}\right\|_{1}}=\frac{2}{\left\|\phi_{0}\right\|_{1}} .
$$

Therefore, Theorem 4.2 is then a direct consequence of Lemma 4.1 and of Theorem 4.2 .5 in [4].

We are now in a position to define the spectral approximation of $\left(v, \phi_{s}\right)$ defined in (4.1)-(4.2). For $1 \leq|m| \leq \ell \leq M$, let $\left\{v_{c, N}^{\ell, m}, v_{g, N}^{\ell, m}, \phi_{s, N}^{\ell, m}\right\}$ be the spectral-Galerkin approximation (as defined in (4.9) and (4.16) with $\omega \equiv 1$ ) of $\left\{v_{c}^{\ell, m}, v_{g}^{\ell, m}, \phi_{s}^{\ell, m}\right\}$ in (4.5)-(4.6). We define the approximation of $\left(v, \phi_{s}\right)$ as follows:

$$
\left\{\begin{array}{l}
v_{N, M}=\sum_{\ell=1}^{M} \sum_{|m| \leq \ell} v_{c, N}^{\ell, m}(\gamma) C_{\ell, m}(\theta, \varphi)+v_{g, N}^{\ell, m}(\gamma) G_{\ell, m}(\theta, \varphi), \\
\phi_{N, M}^{s}=\sum_{\ell=1}^{M} \sum_{|m| \leq \ell} \phi_{s, N}^{\ell, m} Y_{\ell, m}(\theta, \varphi) .
\end{array}\right.
$$

The following result indicates that our approximation is spectrally accurate and has optimal convergence rate.

THEOREM 4.3. Let $\left\{v, \phi_{s}\right\}$ be the solution of (4.1)-(4.2) and $\left\{v_{N, M}, \phi_{N, M}^{s}\right\}$ be the spectral approximation defined in (4.22). Then

$$
\begin{aligned}
\left\|v-v_{N, M}\right\|_{H^{1}(\mathcal{D})} & +\left\|\phi_{s}-\phi_{N, M}^{s}\right\|_{L^{2}\left(S^{2}\right)} \\
& \lesssim\left(N^{1-s}+M^{1-s}\right)\left(\|v\|_{H^{s}(\mathcal{D})}+\left\|\phi_{s}\right\|_{H^{s-1}\left(S^{2}\right)}\right) \\
& \lesssim\left(N^{1-s}+M^{1-s}\right)\left(\|f\|_{H^{s-2}(\mathcal{D})}+\left\|v_{s}\right\|_{H^{s-1}\left(S^{2}\right)}\right)
\end{aligned}
$$


where $D=S^{2} \times(-1,1)$.

Proof. Let $\psi=\sum_{\ell=1}^{\infty} \sum_{|m| \leq \ell}\left(\psi_{c}^{\ell, m} C_{\ell, m}(\theta, \varphi)+\psi_{g}^{\ell, m} G_{\ell, m}(\theta, \varphi)\right)$ be a function on the unit sphere $S^{2}$. We define the orthogonal projector $P_{M}^{s}$ by

$$
P_{M}^{s} \psi=\sum_{\ell=1}^{M} \sum_{|m| \leq \ell}\left(\psi_{c}^{\ell, m} C_{\ell, m}(\theta, \varphi)+\psi_{g}^{\ell, m} G_{\ell m}(\theta, \varphi)\right) .
$$

It can be shown by using a standard procedure (see [7]) that

$$
\left\|\psi-p_{M}^{s} \psi\right\|_{H^{\nu}\left(S^{2}\right)} \lesssim M^{\nu-s}\|\psi\|_{H^{s}\left(S^{2}\right)} \quad \text { for } 0 \leq \nu \leq 1 \leq s .
$$

Now we write the solution $v$ of (4.1) as

$$
v(\gamma, \theta, \varphi)=\sum_{j, \ell=1}^{\infty} \sum_{|m| \leq \ell}\left(v_{c}^{j, \ell, m} C_{\ell, m}(\theta, \varphi) \phi_{j}(\gamma)+v_{g}^{j, \ell, m} G_{\ell, m}(\theta, \varphi) \phi_{j}(\gamma)\right)
$$

where $\phi_{j}(\gamma)=p_{j}(\gamma)+a_{j} p_{j+1}(\gamma)+b_{j} p_{j+2}(\gamma)$ satisfies the boundary conditions (4.2). We then define the projection operator $\Pi_{N, M}$ by

$$
\Pi_{N, M} v=\sum_{j=0}^{N} \sum_{\ell=1}^{M} \sum_{|m| \leq \ell}\left(v_{c}^{j, \ell, m} C_{\ell, m}(\theta, \varphi)+v_{g}^{j, \ell, m} G_{\ell, m}(\theta, \varphi)\right) \phi_{j}(\gamma) .
$$

By using Lemma 4.1 and (4.23), we have

$$
\left\|v-\Pi_{N, M} v\right\|_{H^{\nu}(\Omega)} \lesssim\left(N^{\nu-s}+M^{\nu-s}\right)\|v\|_{H^{s}(\Omega)}, \quad 0 \leq \nu \leq 1 \leq s .
$$

Theorem 4.3 is then a consequence of the above estimates and the following regularity result for (4.1)-(4.2) (cf. [14]):

$$
\|v\|_{H^{s}(\Omega)}+\left\|\phi_{s}\right\|_{H^{s-1}\left(S^{2}\right)} \lesssim\|f\|_{H^{s-2}(\Omega)}+\left\|v_{s}\right\|_{H^{s-1}\left(S^{2}\right)} .
$$

4.2. Fast spectral-Galerkin solvers. According to the above presentation, we have only to solve a sequence of one-dimensional equations (4.5) and (4.6) at each time step. We present below two fast solvers with optimal computational complexity for these equations.

We consider first the spectral-Galerkin approximation (4.9) for (4.7).

Let $p_{i}(x)$ be either the Legendre or Chebyshev polynomial; we set

$$
\phi_{i}(x)=p_{i}(x)+a_{i} p_{i+1}(x)+b_{i} p_{i+2}(x), i=0,1,2, \ldots
$$

such that

$$
a_{ \pm} \phi_{i}( \pm 1)+b_{ \pm} \phi_{i}^{\prime}( \pm 1)=0 .
$$

It is shown in [24] that $\left\{a_{i}, b_{i}\right\}$ in (4.24) can be determined uniquely. Therefore, we have

$$
X_{N}=\operatorname{span}\left\{\phi_{0}, \phi_{1}, \ldots, \phi_{N-2}\right\}
$$


Setting

$$
\begin{aligned}
& a_{i j}=-\left(\phi_{j}^{\prime \prime}, \phi_{i}\right)_{w}, \quad A=\left(a_{i j}\right), u_{N}=\sum_{i=0}^{N-2} x_{i} \phi_{i}(x), \\
& b_{i j}=\left(\phi_{j}, \phi_{i}\right)_{w}, B=\left(b_{i j}\right), \quad f_{i}=\left(I_{N} f, \phi_{i}\right)_{w}, \\
& \bar{x}=\left(x_{0}, x_{1}, \ldots, x_{N-2}\right)^{T}, \quad \bar{f}=\left(f_{0}, f_{1}, \ldots, f_{N-2}\right)^{T},
\end{aligned}
$$

then (4.9) (with $f$ replaced by $I_{N} f$ ) is equivalent to the linear system

$$
(\alpha B+A) \bar{x}=\bar{f} .
$$

Thanks to the orthogonal properties of Legendre and Chebyshev polynomials, we see that $B$ is symmetric and has only five nonzero diagonals. We recall that in the Legendre case $A$ is diagonal [22], and in the Chebyshev case $A$ is a special upper-triangular matrix such that (4.26) can be solved in $\mathcal{O}(N)$ operations [23].

The equations in (4.16) can be treated in a similar fashion. Indeed, consider first the Legendre case $(w \equiv 1)$. Setting

$$
Y_{N}=\operatorname{span}\left\{\phi_{1}, \phi_{2}, \ldots, \phi_{N-2}\right\}
$$

then we have

$$
\int_{-1}^{1} u(x) d x=0 \forall u \in Y_{N}
$$

Therefore, let

$$
\widetilde{A}=\left(a_{i j}\right)_{i, j=1, \ldots, N-2}, \widetilde{B}=\left(b_{i j}\right)_{i, j=1, \ldots, N-2}
$$

and

$$
\bar{x}=\left(x_{1}, x_{2}, \ldots, x_{N-2}\right)^{T}, \quad \bar{f}=\left(f_{1}, f_{2}, \ldots, f_{N-2}\right)^{T},
$$

where $a_{i j}, b_{i j}, x_{i}, f_{i}$ are defined in (4.25). Then, (4.16) (with $\omega \equiv 1$ and $f$ replaced by $\left.I_{N} f\right)$ reduces to the following linear system:

$$
(\alpha \widetilde{B}+\widetilde{A}) \widetilde{x}=\widetilde{f},
$$

which can be solved efficiently since $\widetilde{A}$ is diagonal and $\widetilde{B}$ is penta-diagonal.

In the Chebyshev case, (4.16) cannot be easily decoupled so we will solve it as a coupled system. Setting

$$
\begin{aligned}
u_{N}=\sum_{i=0}^{N-2} x_{i} \phi_{i}(x), & w_{i}=\int_{-1}^{1} \phi_{i}(x) d x, \\
\bar{w}=\left(w_{0}, w_{1}, \ldots, w_{N-2}\right)^{T}, & \bar{h}=(2,0, \ldots, 0)^{T}, \\
\hat{x}=\left(x_{0}, x_{1}, \ldots, x_{N-2}, \gamma_{N}\right)^{T}, & \hat{f}=\left(f_{0}, f_{1}, \ldots, f_{N-2}, 0\right)^{T},
\end{aligned}
$$

then (4.16) is equivalent to

$$
\left[\begin{array}{cc}
\alpha B+A & \bar{h} \\
\bar{w}^{T} & 0
\end{array}\right] \hat{x}=\hat{f} .
$$

Since $(\alpha B+A) \bar{x}=\bar{f}$ can be solved in $\mathcal{O}(N)$ operations by a special Gaussian elimination process, we see that (4.28) can also be solved in $\mathcal{O}(N)$ operations by the same process. 
5. Concluding remarks. We have presented a fast and accurate numerical method for the approximation of the primitive equations of the atmosphere with simplified model physics. The method was based on a special semi-implicit time discretization scheme which requires us to solve a Helmholtz equation and the nonlocal Stokes problem with constant coefficients at each time step. Thanks to the orthogonal properties of vectorial spherical harmonics, we were able to decompose the nonlocal Stokes problem (and the Helmholtz equation) into a sequence of one-dimensional equations in the vertical variable. We then proposed a fast spectral-Galerkin method using Legendre or Chebyshev polynomials for solving these one-dimensional equations with optimal computational complexity. We emphasize that this scheme is extremely efficient since we need only to deal with, at each time step, linear systems which can be solved by a direct (without iterations) method with optimal computational complexity. A drawback of this scheme is that the use of spherical harmonics entails expensive transformations which are needed for computing the nonlinear terms with the transform method $[19,17]$. However, this situation may be improved with the recent contribution of [11] on accelerating spherical harmonics transforms.

In addition to the superior efficiency and accuracy of the spatial approximation, the fully discretized scheme is unconditionally stable from a numerical analysis point of view for fixed physical parameters, thanks to the implicit treatment of the linear elliptic terms in the PEs. We would like to point out that this unconditional stability should be viewed with caution in practical situations, for the scheme is only stable for $\Delta t$ less than a certain critical time step $\Delta t_{c}$ which is related to the physical parameters. However, it does imply in particular that grid refinement can be performed without reducing the time step.

The construction of a fast and accurate numerical scheme is only a first step toward our long-range objective of better understanding the phenomena of atmosphere and climate. The scheme is currently being implemented to simulate various atmospheric phenomena including, in particular, the interannual variability of the global atmosphere; numerical results and their interpretations, which are beyond the scope of this paper, will be presented elsewhere.

Appendix A. Nondimensionalization of the PEs. In this section, we briefly describe the derivation of the nondimensional PEs (2.1)-(2.5) from the PEs below which are given in a $p$-coordinate system $(\theta, \varphi, p)$, where $\theta$ is the colatitude $(0 \leq \theta \leq$ $\pi), \varphi$ is the longitude $(0 \leq \varphi \leq 2 \pi)$, and $p$ is the pressure (see $[14,21,28])$ :

$$
\left\{\begin{array}{l}
\frac{\partial v}{\partial t}+\nabla_{v} v+\omega \frac{\partial v}{\partial p}+2 \Omega \cos \theta k \times v+\nabla \phi+\widetilde{L}_{1} v=0 \\
\frac{\partial \phi}{\partial p}+\frac{R T}{p}=0 \\
\frac{R^{2}}{c^{2}}\left[\frac{\partial T}{\partial t}+\nabla_{v} T+\omega \frac{\partial T}{\partial p}\right]-\frac{R \omega}{p}+\widetilde{L}_{2} T=\widetilde{Q} \\
\operatorname{div} v+\frac{\partial \omega}{\partial p}=0 .
\end{array}\right.
$$

- The space domain is given by

$$
\widetilde{M}=\widetilde{S}^{2} \times\left(p_{0}, P\right),
$$

where $\widetilde{S}^{2}$ is the two-dimensional sphere with radius $a$, representing the surface of the earth, $p_{0}$ is the pressure on top of the atmosphere, and $P$ is the pressure 
on the surface of the earth. We assume that $P$ and $p_{0}$ are constants. The positive constant $R$ is the gas constant, $g$ the gravitational constant, and $\Omega$ the angular velocity of the earth.

- The unknown functions are the horizontal wind velocity $v$, the vertical velocity $\omega$ in the pressure coordinates, the temperature function $T$, and the geopotential $\phi$.

- The linear differential operators $\widetilde{L}_{1}$ and $\widetilde{L}_{2}$ are defined by

$$
\begin{aligned}
& \widetilde{L}_{1} v=-\mu_{1} \Delta v-\nu_{1} \frac{\partial}{\partial p}\left(\left(\frac{g p}{R \bar{T}}\right)^{2} \frac{\partial v}{\partial p}\right), \\
& \widetilde{L}_{2} T=-\mu_{2} \Delta T-\nu_{2} \frac{\partial}{\partial p}\left(\left(\frac{g p}{R \bar{T}}\right)^{2} \frac{\partial T}{\partial p}\right),
\end{aligned}
$$

where $\mu_{i}$ and $\nu_{i}(i=1,2)$ are (positive) viscosity coefficients.

- The operators $\nabla, \nabla_{v}, \Delta$ are horizontal differential operators in spherical coordinates on $\widetilde{S}^{2}$.

- The given function $\bar{T} \in C^{\infty}\left(\left[p_{0}, P\right]\right)$ is determined by

$$
c^{2}=R\left(\frac{R \bar{T}}{c_{p}}-p \frac{\partial \bar{T}}{\partial p}\right)=\text { const. }
$$

The function $\bar{T}$ can be considered as the mean profile of the temperature function averaged on the isobaric surface, and the constant $c$ is a stability constant. We refer to $[14,15]$ for more detailed explanations about $\bar{T}$ and $c^{2}$.

The boundary conditions for the PEs are given by

$$
\left\{\begin{array}{l}
\frac{\partial v}{\partial p}=-\widetilde{\gamma}_{s} v, \omega=0, \quad \frac{\partial T}{\partial p}=\widetilde{\alpha}_{s}\left(\widetilde{T}_{s}-T\right) \quad \text { at } p=P \\
\frac{\partial v}{\partial p}=0, \omega=0, \frac{\partial T}{\partial p}=0 \quad \text { at } p=p_{0} .
\end{array}\right.
$$

We consider the typical horizontal length scale $L=a$ and a reference horizontal velocity $U$, and we set

$$
\left\{\begin{array}{l}
v=v^{\prime} U, \quad \omega=\frac{\left(P-p_{0}\right) U}{a} \omega^{\prime}, \quad T=\frac{a U \Omega}{R} T^{\prime}, \\
\phi=a U \Omega \phi^{\prime}, \\
t=\frac{a}{U} t^{\prime}, \quad p=P-\left(P-p_{0}\right) \eta,
\end{array}\right.
$$




$$
\left\{\begin{array}{l}
f=2 \cos \theta, \quad R o=\frac{U}{a \Omega}, \\
\alpha=\frac{a^{2} \Omega^{2}}{c^{2}}, \quad Q=\frac{\widetilde{Q} a^{2} \Omega}{U^{2} R}, \\
\frac{1}{R e_{1}}=\frac{\mu_{1}}{a U}, \quad \frac{1}{R e_{2}}=\frac{P^{2} \nu_{1} a g^{2}}{\left(P-p_{0}\right)^{2} U R^{2} \bar{T}_{0}^{2}}, \\
\frac{1}{R t_{1}}=\frac{\mu_{2} a \Omega^{2}}{U R^{2}}, \quad \frac{1}{R t_{2}}=\left(\frac{P}{P-p_{0}}\right)^{2} \frac{a^{3} \Omega^{2} \nu_{2} g^{2}}{\bar{T}_{0}^{2} R^{2} U,}, \\
K_{2}(\eta)=\frac{P}{P-p_{0}}-\eta, \quad K_{1}(\eta)=\left(\frac{p \bar{T}_{0}}{P \bar{T}}\right)^{2}, \\
\bar{T}_{0}=\frac{a U \Omega}{R} .
\end{array}\right.
$$

Substituting (A.4)-(A.5) into (A.1) and dropping all primes in the resulting equations, we obtain the following nondimensional PEs in the nondimensional pseudospatial domain $M=S^{2} \times(0,1)$ :

$$
\begin{aligned}
& {\left[\frac{\partial v}{\partial t}+\nabla_{v} v-\omega \frac{\partial v}{\partial \eta}\right]+\frac{1}{R o}[f k \times v+\nabla \phi]+L_{1} v=0,} \\
& \alpha\left[\frac{\partial T}{\partial t}+\nabla_{v} T-\omega \frac{\partial T}{\partial \eta}\right]-\frac{1}{R o} \frac{\omega}{K_{2}}+L_{2} T=Q, \\
& \operatorname{div} v-\frac{\partial \omega}{\partial \eta}=0, \\
& \frac{\partial \phi}{\partial \eta}=\frac{T}{K_{2}}
\end{aligned}
$$

where $L_{1}$ and $L_{2}$ are defined in (2.7). The nondimensional boundary conditions are

$$
\left\{\begin{array}{l}
\frac{\partial v}{\partial \eta}=\gamma_{s} v, \quad \omega=0, \quad \frac{\partial T}{\partial \eta}=\alpha_{s}\left(T-T_{s}\right) \text { at } \eta=0, \\
\frac{\partial v}{\partial \eta}=0, \quad \omega=0, \quad \frac{\partial T}{\partial \eta}=0 \text { at } \eta=1 .
\end{array}\right.
$$

Here $\alpha_{s}, \gamma_{s}, v_{s}, T_{s}$ are the nondimensional forms of $\widetilde{\alpha}_{s}, \widetilde{\gamma}_{s}, \widetilde{v}_{s}$, and $\widetilde{T}_{s}$. Integrating the diagnostic equations (A.8)-(A.9) and taking into account (2.5) $(\omega=0$ at $\eta=1)$, we find

$$
\left\{\begin{array}{l}
\omega=W(v)=-\operatorname{div} \mathcal{M}^{*} v \\
\operatorname{div} \int_{0}^{1} v d \eta=0 \\
\phi=\phi_{s}+\mathcal{M}\left(\frac{T}{K_{2}}\right)
\end{array}\right.
$$

where $\mathcal{M}$ and its adjoint (in the $L^{2}$ sense) $\mathcal{M}^{*}$ are defined in (2.6). In (A.11), the function $\phi_{s}$, depending only on $\theta$ and $\varphi$, is the unknown value of $\phi=g z$ at the isobar $p=P(\eta=0)$. We then obtain (2.1)-(2.3) by substituting (A.11) into (A.6)-(A.9). 


\section{Appendix B. Spherical harmonics.}

B.1. Scalar spherical harmonics. We first recall the definition of scalar spherical harmonics. Readers are referred to [8] or [18] for more details. For any $\ell=0,1, \ldots$, and $0 \leq m \leq \ell$, we define

$$
\begin{gathered}
Y_{\ell, m}(\theta, \varphi)=\Theta_{\ell, m}(\theta) e^{i m \varphi} \\
\Theta_{\ell, m}(\theta)=\left[\frac{(2 \ell+1)(\ell-m) !}{4 \pi(\ell+m) !}\right]^{1 / 2} P_{\ell, m}(\cos \theta),
\end{gathered}
$$

where $P_{\ell, m}$ is the associated Legendre function defined by

$$
P_{\ell, m}(\mu)=(-1)^{m}\left(1-\mu^{2}\right)^{m / 2} \frac{d^{m}}{d \mu^{m}} P_{\ell}(\mu) .
$$

Here $P_{\ell}(\mu)$ is the $\ell$ th Legendre polynomial. Then we define $Y_{\ell,-m}(\theta, \varphi)(m>0)$ by

$$
Y_{\ell,-m}(\theta, \varphi)=(-1)^{m}\left[Y_{\ell, m}(\theta, \varphi)\right]^{*},(* \text { being the conjugate transpose operator }) .
$$

Here are some basic properties of the scalar spherical harmonics:

- $\left\{Y_{\ell, m}|\ell=0,1, \ldots| m \mid, \leq \ell\right\}$ forms an orthonormal basis of $L^{2}\left(S^{2}\right)$. Consequently, for all $f \in L^{2}\left(S^{2}\right)$, we have

$$
\left\{\begin{array}{l}
f(\theta, \varphi)=\sum_{\ell=0}^{\infty} \sum_{|m| \leq \ell} f^{\ell, m} Y_{\ell, m}(\theta, \varphi), \\
f^{\ell,-m}=(-1)^{m}\left(f^{\ell, m}\right)^{*}, \\
f^{\ell, m} \in \mathbb{C}^{1},
\end{array}\right.
$$

and the $L^{2}$-norm of $f$ is given by

$$
|f|=\left(\sum_{\ell=0}^{\infty} \sum_{|m| \leq \ell}\left|f^{\ell, m}\right|^{2}\right)^{1 / 2} .
$$

- $\left\{Y_{\ell, m}|\ell=0,1, \ldots| m \mid, \leq \ell\right\}$ are complex eigenfunctions of the LaplaceBeltrami operator on $S^{2}$, i.e.,

$$
\left\{\begin{array}{r}
-\Delta Y_{\ell, m}=\lambda_{\ell} Y_{\ell, m}, \\
\lambda_{\ell}=\ell(\ell+1) .
\end{array}\right.
$$

Therefore it is evident that for any $f$ with expansion (B.2) and sufficient regularity,

$$
\left|(-\Delta)^{\alpha} f\right|^{2}=\sum_{\ell=0}^{\infty} \sum_{|m| \leq \ell} \lambda_{\ell}^{2 \alpha}\left|f^{\ell, m}\right|^{2} .
$$

The eigenvalue $\lambda_{\ell}$ has multiplicity $2 l+1$. 
B.2. Vectorial spherical harmonics. For any $\ell=0,1, \ldots$, and $|m| \leq \ell$, we define

$$
\left\{\begin{array}{l}
G_{\ell, m}=\lambda_{\ell}^{-1 / 2} \nabla Y_{\ell, m} \\
C_{\ell, m}=\lambda_{\ell}^{-1 / 2} \operatorname{curl} Y_{\ell, m}
\end{array}\right.
$$

By the Hodge decomposition, for any $s \in \mathbb{R}^{1}$, we have

$$
H^{s}\left(T S^{2}\right)=\nabla H^{s+1}\left(S^{2}\right) \oplus \operatorname{curl} H^{s+1}\left(S^{2}\right) .
$$

Hence it is easy to see that (cf. [16]) $\left\{G_{\ell, m}, C_{\ell, m}|\ell=0,1, \ldots| m \mid, \leq \ell\right\}$ forms an orthonormal basis of $L^{2}\left(T S^{2}\right)$. In particular, for any vector field $v \in L^{2}\left(T S^{2}\right)$, we have

$$
\left\{\begin{array}{l}
v=\sum_{\ell=0}^{\infty} \sum_{|m| \leq \ell}\left[v_{g}^{\ell, m} G_{\ell, m}+v_{c}^{\ell, m} C_{\ell, m}\right], \\
v_{g}^{\ell,-m}=(-1)^{m}\left(v_{g}^{\ell, m}\right)^{*}, \quad v_{c}^{\ell,-m}=(-1)^{m}\left(v_{c}^{\ell, m}\right)^{*} .
\end{array}\right.
$$

Moreover, the $L^{2}$-norm of $v$ is given by

$$
|v|^{2}=\sum_{\ell=0}^{\infty} \sum_{|m| \leq \ell}\left[\left|v_{g}^{\ell, m}\right|^{2}+\left|v_{c}^{\ell, m}\right|^{2}\right] .
$$

Furthermore, since the Laplace-Beltrami operators for vectors and functions commute with the gradient and curl operators, it is easy to show that $\left\{G_{\ell, m}, C_{\ell, m} \mid \ell=\right.$ $0,1, \ldots$, and $|m| \leq \ell\}$ are a complete set of eigenfunctions of the Laplacian. Namely,

$$
\left\{\begin{aligned}
-\Delta G_{\ell, m} & =\lambda_{\ell} G_{\ell, m}, \\
-\Delta C_{\ell, m} & =\lambda_{\ell} C_{\ell, m} .
\end{aligned}\right.
$$

For any $v \in \mathcal{D}\left((-\Delta)^{\alpha}\right)$, we also have

$$
\left|(-\Delta)^{\alpha} v\right|_{L^{2}}^{2}=\sum_{\ell=0}^{\infty} \sum_{|m| \leq \ell} \lambda_{m}^{2 \alpha}\left[\left|v_{g}^{\ell, m}\right|^{2}+\left|v_{c}^{\ell, m}\right|^{2}\right] .
$$

Note that the definition (B.3) is used in [16] and that $\left\{G_{\ell, m}, C_{\ell, m}\right\}$ are in fact the horizontal components of the standard vectorial spherical harmonics.

Acknowledgment. The authors would like to thank the Isaac Newton Institute for Mathematical Sciences at Cambridge University for their hospitality.

\section{REFERENCES}

[1] B. K. Alpert and V. Rokhlin, A fast algorithm for the evaluation of Legendre expansions, SIAM J. Sci. Stat. Comput., 12 (1991), pp. 158-179.

[2] C. Bernardi, C. Canuto, and Y. Maday, Generalized inf-sup conditions for Chebyshev spectral approximation of the Stokes problem, SIAM J. Numer. Anal., 25 (1988), pp. 1237-1271.

[3] C. Bernardi and Y. Maday, Properties of some weighted Sobolev spaces and applications to spectral approximations, SIAM J. Numer. Anal., 26 (1989), pp. 769-829.

[4] C. Bernardi And Y. Maday, Approximations Spectrales de Problèmes aux Limites Elliptiques, Springer-Verlag, Paris, 1992. 
[5] C. Canuto, M. Y. Hussaini, A. Quarteroni, and T. A. Zang, Spectral Methods in Fluid Dynamics, Springer-Verlag, New York, 1987.

[6] C. Canuto and A. Quarteroni, Spectral and pseudospectral methods for parabolic problems with nonperiodic boundary conditions, Calcolo, 18 (1981), pp. 197-217.

[7] C. Canuto and A. Quarteroni, Approximation results for orthogonal polynomials in Sobolev spaces, Math. Comp., 38 (1982), pp. 67-86.

[8] R. Courant and D. Hilbert, Methods of Mathematical Physics, Vol. 1, Wiley Interscience, New York, 1953.

[9] R. DALEY, The development of efficient time integration schemes using model normal modes, Monthly Weather Rev., 108 (1980), pp. 100-110.

[10] R. Daley, C. Girard, J. Henderson, And I. Simmonds, Short-term forecasting with a multilevel spectral primitive equation model. part $i$ - model formulation, Atmosphere, 14 (1976), pp. 98-116.

[11] J. R. Driscoll and D. M. Healy, Computing Fourier transforms and convolutions on the 2-sphere, Adv. in Appl. Math., 15 (1994), pp. 202-250.

[12] B. J. Hoskins And A. J. Simmons, A multi-layer spectral model and the semi-implicit method, Quart. J. Roy. Met. Soc., 101 (1975), pp. 637-655.

[13] M. JARRAUD AND A. P. M. BAEDE, The use of spectral techniques in numerical weather prediction, in Large Scale Computations in Fluid Mechanics, B. Enquist, S. Osher, and R. Somerville, eds., Lectures in Appl. Math. 22, AMS, Washington, DC, 1985, pp. 1-41.

[14] J. L. Lions, R. Temam, And S. WAng, New formulations of the primitive equations of the atmosphere and applications, Nonlinearity, 5 (1992), pp. 237-288.

[15] J. L. Lions, R. Temam, And S. Wang, Models of the coupled atmosphere and ocean (CAO I), Comput. Mech. Adv., 1 (1993), pp. 3-54.

[16] J. L. Lions, R. Temam, And S. WAnG, Numerical analysis of the coupled atmosphere and ocean models (CAO II), Comput. Mech. Adv., 1 (1993), pp. 55-120.

[17] B. Machenhauer and R. Daley, A Baroclinic Primitive Equation Model with a Spectral Representation in 3-Dimensions, Tech. report 4, Institut for Teoretisk Meteorologi, Kobenhavns Universitet, Copenhagen, Denmark, 1972.

[18] P. M. Morse And H. Feshback, Methods of Theoretical Physics, McGraw-Hill, New York, 1953.

[19] S. A. Orszag, Transform method for calculation of vector coupled sums: Applications to the spectral form of the vorticity equation, J. Atmospheric Sci., 27 (1970), pp. 890-895.

[20] A. RoBert, A stable numerical integration scheme for the primitive meteorological equations, Atmosphere-Oceans, 19 (1981), pp. 35-46.

[21] M. L. Salby, Fundamentals of Atmospheric Physics, Academic Press, New York, 1996.

[22] J. SHEN, Efficient spectral-Galerkin method I. Direct solvers for second- and fourth-order equations using Legendre polynomials, SIAM J. Sci. Comput., 15 (1994), pp. 1489-1505.

[23] J. SHEn, Efficient spectral-Galerkin method II. Direct solvers for second- and fourth-order equations using Chebyshev polynomials, SIAM J. Sci. Comput., 16 (1995), pp. 74-87.

[24] J. SHEN, Efficient Chebyshev-Legendre Galerkin methods for elliptic problems, in Proc. Internat. Conf. Spectral and High Order Methods (ICOSAHOM '95), Houston J. Math., A. V. Ilin and R. Scott, eds., 1996, pp. 233-240.

[25] A. J. Simmons, B. J. Hoskins, and D. M. Burridge, Stability of the semi-implicit method of time integration, Monthly Weather Rev., 106 (1978), pp. 405-412.

[26] K. E. Trenberth, Climate System Modelling, Cambridge University Press, Cambridge, 1993.

[27] S. Wang, On Solvability for the Equations of the Large-Scale Atmospheric Motion, Ph.D. thesis, Lanzhou University, China, 1988.

[28] W. M. Washington and C. L. Parkinson, An Introduction to Three-Dimensional Climate Modeling, Oxford University Press, Oxford, 1986. 